\title{
USING SEMANTIC WEB AND ONTOLOGIES TO CREATE INTELLIGENT AND PERSONALIZED SYSTEMS FOR LEARNING PURPOSES
}

\author{
Roopesh Kevin Sungkur ${ }^{1 \star}$, Ibne Raheeman, Yusrah Musbally \\ ${ }^{1}$ Computer Science and Engineering Dept, University of Mauritius \\ ${ }^{*}$ Corresponding author
}

\begin{abstract}
There was a time when it was thought that the more information someone has, the better it is. Nowadays, there has been a change in the mindset of people where what they want is meaningful and personalized information. The semantic web is an advancement of the current web that is being used, the web 2.0, which comprises mainly of metadata i.e. data about data. The semantic web is a new technology which is being developed and it is seen as the future web where everything will be much more accurate as per our needs. Semantic Web technologies and applications are getting increasingly popular and adopted in different fields, including education. Research on software and education has already shown some of the features expected to be embedded in the next generation of learning support systems. Such features include: more adaptive and personalized learning environment, a better use of pedagogies to enhance instruction/learning, effective information sharing, storage and retrieval, new forms of collaboration with peers, and many other characteristics that enable the realization of AAAL: Anytime, Anywhere, Anybody Learning. Information on the Web is commonly represented in natural-language for human understanding. However, in order for the computer to understand its meaning, it is necessary to represent the information in a form that can be interpreted syntactically and semantically. Such representation helps the process of analyzing, extracting, and integrating information on the Web, making it easier the creation of solid knowledge bases that intelligent services can rely on to support users' needs. Nowadays, research on ontologies has been considered as one of the keys to provide information in a computer-understandable way. This paper focuses on the different Semantic Web Technologies that play an important role in education and learning.
\end{abstract}

Keywords: Semantic Web, Personalized Learning, Intelligent Systems, Ontologies, Education

\section{INTRODUCTION}

The Semantic Web is a "web of data" that facilitates machines to understand the semantics, or meaning, of information on the World Wide Web. Semantic Web is imagined as a system that allows computers to understand and respond to complex human queries. Computers will be better able to search, process, integrate and present the content of these resources in a meaningful and intelligent manner.

\subsection{Evolution of the Web}

Over the recent years, the World Wide Web has rapidly evolved from a small computer science project to an international collaboration of global networks that drives many of the world's markets. The www has undergone many evolutions to respond to the rapid changes in technologies and people's needs. 


\section{Web 1.0 (the "Information Web"):}

The original World Wide Web has often been called Web 1.0 since the coming of Web 2.0. Web-1.0 pages are for humans to read and understand. There is no obligation of machine processability. On Web-1.0 pages, the majority of hyperlinks are manually assigned by webmasters. Web-1.0 pages contain only reactive functions or services. [1]

\section{Web 2.0 (the "Social Web"):}

The web 1.0 evolved to become web 2.0 after some time when more users started using the www and it had more success than the 1.0. The success of Web 2.0 lies on the success of two technologies: blogging and tagging. Essentially, blogging enhances the character of content, and tagging enhances the character of link. The blogging technology extends the update of content from personal activities to social activities. The tagging technology enables the creation of hyperlinks from tedious, individual behaviors to handy, collaborative behaviors. [2]

\section{Web 3.0 (the "Semantic Web")}

The Semantic Web can be thought of as an extension of the present web. The goal of Semantic Web enables machine-processable content and automatic machine processing so that machines can understand web content. Semantic annotation and authoring on Semantic Web allow content in web pages to bemapped to formal ontology definitions, which are machine-processable. The Semantic Web is emerging, and is characterized by:

- Linked data or hyperdata, where data objects are linked to other data objects (similar to how web pages are linked today)

- Large hyperdata datasets such as DBpedia (a community effort to extract structured information from Wikipedia and make the information available on the Web)

- A query language for hyperdata capable of treating the entire web as a single datastore, called SPARQL. [3]

Its search engines retrieve micro content texts which were tagged automatically. It translates billions of Web 1.0 macro contents into micro contents. Precise search results would be available because tagging can solve part of the ambiguity that homonyms and synonyms introduce into the process of search. The Web makes use of a declarative ontological language like OWL to produce domain-specific ontologies that machines can use to reason about information and make new conclusions, not simply match keywords.

\subsection{E-Learning}

E-learning is now one of the main forms of learning. According to research, around 35 million students worldwide make use of e-learning. Most of the learning is web 2.0 as this is the most stable technology currently. According to a pedagogical research, interactions between students help for better learning and this is why e-learning has put greater emphasis on social learning and social software such as blogs, wikis and virtual worlds. Some advanced universities have started to introduce semantic in their e-learning website but it is in its infancy. Semantics has been introduced in some e-learning to improve searching.

\subsection{Goals of the Semantic Web in the educational field}

Current websites are universal, scalable, decentralized and evolvable. The overall objective of Semantic Web techniques is building online end-user applications that can integrate, combine and deduce information to assist users in performing their tasks more efficiently. Below are some main goals of a semantic web application in general. The application has to be an end-user application, i.e. an application that provides a practical value to general Web users. The information sources used should be under diverse ownership or control should be heterogeneous (syntactically, structurally, and semantically) $[4,5,6]$

- The meaning of data has to play a central role. Data must be manipulated/processed in interesting ways to derive useful information and it should achieve things that alternative technologies cannot do [7]

- Web of data provides common data representation framework to facilitate integrating multiple sources to draw new conclusions

- Increase the utility of information by connecting it to its definitions and to its context and more efficient information access and analysis [8] 


\section{LITERATURE REVIEW}

\subsection{Characteristics of the Semantic Web}

Some of the most important characteristics of the Semantic Web include Data Portability, Ubiquity, Dynamic Content, Possibility of Inferences and Rich User Experience. A brief description of these characteristics is given below.

Table 1: Characteristics of the Semantic Web [9]

\begin{tabular}{|l|l|}
\hline Data Portability & $\begin{array}{l}\text { Use common agreed upon standards to markup information, so that they can } \\
\text { be mashed up in contexts the original data provider did not anticipate. This } \\
\text { means that an object in a data space should be movable and should also have } \\
\text { the ability to be referenced using an identifier such as a URI. }\end{array}$ \\
\hline Ubiquity & $\begin{array}{l}\text { Make the above marked up data available in the widest possible channels. } \\
\text { HTTP and the REST route should be the protocol; XMPP is another delivery } \\
\text { channel, if data is time-sensitive. }\end{array}$ \\
\hline Expose data graph & $\begin{array}{l}\text { Defining a grammar for data via ontology and referencing to some other } \\
\text { element that will enhance or clarify its meaning. Mapping and translating } \\
\text { between ontologies must also be possible. }\end{array}$ \\
\hline Allow inferences & $\begin{array}{l}\text { To make non-obvious connections bare should be the outcome for a data graph } \\
\text { that is linked deeply. To make patterns hidden with data apparent. }\end{array}$ \\
\hline Dynamic content & $\begin{array}{l}\text { Semantic web services have to be dynamic and proactive in all aspects as } \\
\text { automatic searching will be done. }\end{array}$ \\
\hline $\begin{array}{l}\text { Rich user } \\
\text { Experience }\end{array}$ & $\begin{array}{l}\text { technologies for producing rich media have helped making web services lighter, } \\
\text { faster, less cluttered and more interesting to the end user. }\end{array}$ \\
\hline
\end{tabular}

\subsection{Semantic Web Architecture}

The four versions of the Semantic Web reference architecture (V1-V4) proposed by Berners-Lee [10], as presented in Figure 1.
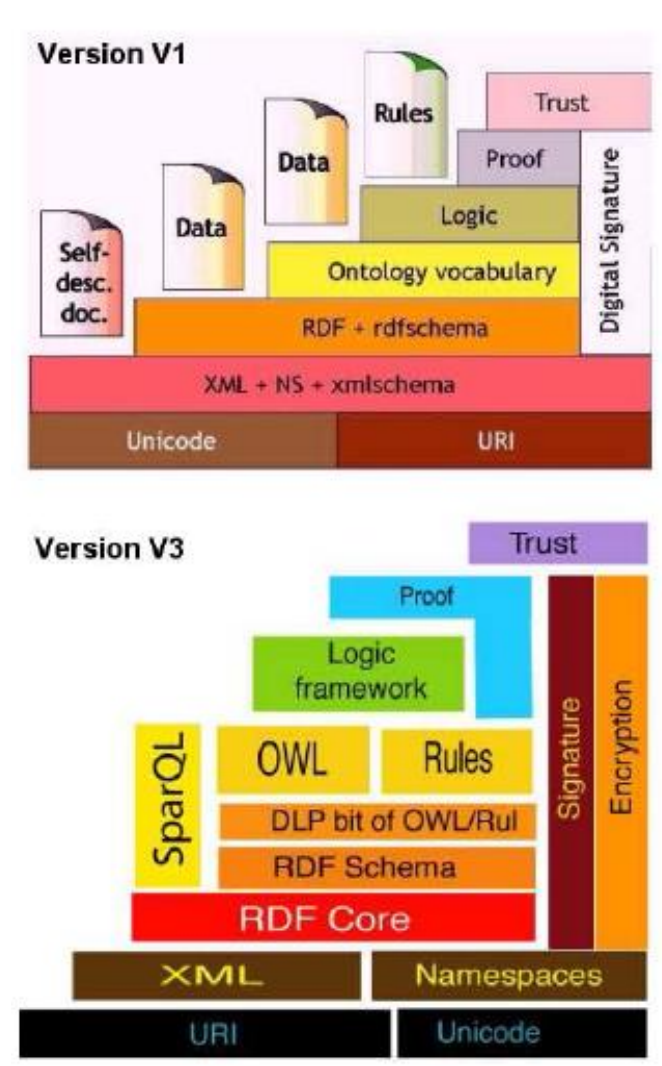

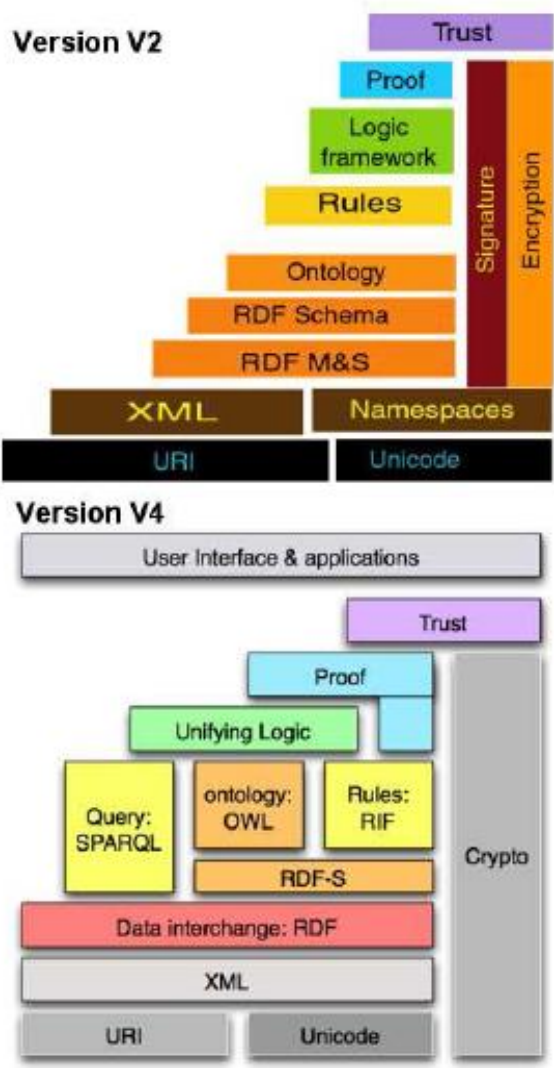

Figure 1: Semantic Web architecture 
Table 2 below describes some of the most important concept to be able to understand Semantic Web Architecture.

Table 2: Concepts of Semantic Web Architecture [11]

\begin{tabular}{|l|l|}
\hline Unicode and URI & $\begin{array}{l}\text { Unicode is the standard for computer character representation designed to help } \\
\text { developers who want to create software applications that work in any language } \\
\text { in the world. URIs, the standard for identifying and locating resources (such as } \\
\text { pages on the Web), provide a baseline for representing characters used in most } \\
\text { of the languages in the world. }\end{array}$ \\
\hline XML & $\begin{array}{l}\text { XML and its related standards, such as Namespaces, and Schemas, form a } \\
\text { common means for structuring data on the Web, but without communicating the } \\
\text { meaning of the data. }\end{array}$ \\
\hline $\begin{array}{l}\text { Resource Description } \\
\text { Framework }\end{array}$ & $\begin{array}{l}\text { RDF is the first layer of the Semantic Web proper. RDF is a standard model for } \\
\text { data interchange on the Web. It is a simple metadata representation framework, } \\
\text { using URIs to identify Web-based resources. A graph view model which is the } \\
\text { easiest possible mental model for RDF and is often used in easy-to-understand } \\
\text { visual explanations is used for describing relationships between resources. }\end{array}$ \\
\hline RDF Schema & $\begin{array}{l}\text { a simple type modeling language for describing classes of resources and } \\
\text { properties between them in the basic RDF model. It provides a simple } \\
\text { reasoning framework for inferring types of resources. }\end{array}$ \\
\hline Ontologies & $\begin{array}{l}\text { a richer language for providing more complex constraints on the types of } \\
\text { resources and their properties. }\end{array}$ \\
\hline Trust & $\begin{array}{l}\text { The final layer of the stack addresses issues of trust that the Semantic Web can } \\
\text { support. Trust is needed on the Semantic Web for content filtering, making } \\
\text { statements about statements, defining security at the semantic level and for } \\
\text { knowledge sharing. }\end{array}$ \\
\hline
\end{tabular}

\subsection{The setting for Semantic Web in the Education field}

Teaching, learning, collaboration, assessment, and other educational activities on the Semantic Web is represented below in Figure 2

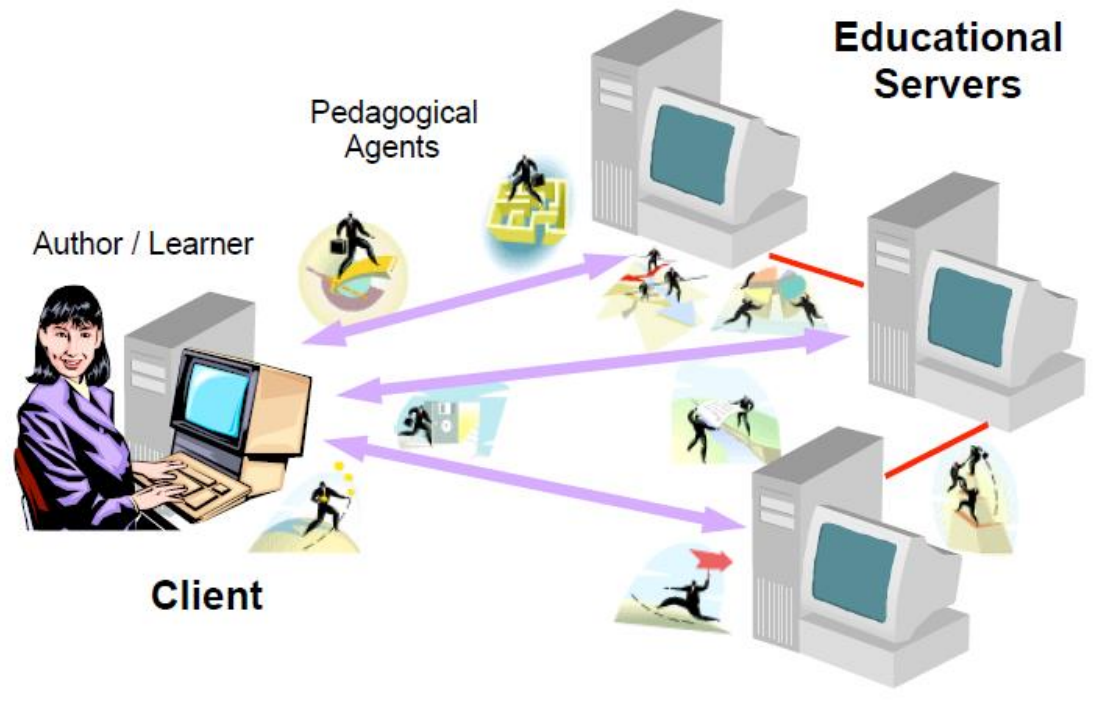

Figure 2: Semantic Web in the educational field [12, 13]

Pedagogical agents are autonomous software entities that support human learning by interacting with students/learners and authors/teachers and by collaborating with other similar agents, in the context of interactive learning environments. Pedagogical agents provide the necessary infrastructure for knowledge and information flow between the clients and the servers. They play an important role as they help in locating, browsing, and selecting, arranging, integrating using educational material from different educational servers. [14] 
There are several types of agents:

1. Tutoring Agents: ensure the interaction between the features of the system and the users according to their roles.

2. Support Agents: support the others agents to ensure the accomplishment of their activities.

3. Controller Agents: verify the performance of the agents and semantic web services; provide access to agents in the system.

From the learner's perspective the server appears to act as an intelligent tutor with both domain and pedagogical knowledge to conduct a learning session.

\subsection{Ontologies}

Ontology is a formal and explicit specification of a shared conceptualization (Gruber, 1993). In this definition, conceptualization refers to an abstract model of a phenomenon in which the relevant concepts are identified; explicit refers to the need for specifying the different concepts that conforms an ontology; formal means that the specification must be represented in a formalized representation language; shared indicates that the ontology describes knowledge accepted by a community[15]. Ontologies have become the de-facto standard knowledge representation technology after the emergence of the Semantic Web along with Semantic Web Services and the Semantic Grid. For all these new research branches, ontologies are the cornerstone technology. In a web context, ontologies provide a shared understanding of a domain. Such sharing is needed to avoid terminological differences [16].

\subsection{Other semantic web available in learning field}

Some semantic webs used for education purposes across the world are found below. It should be noted that all these websites are not completely semantic as no complete semantic web have already been made. These websites are built from web 2.0 technologies and a small part of semantic web integrated in it. This is exactly what we will try to do in this project by introducing the semantic web.

\subsubsection{University of Southampton}

University of Southampton in fact created a semantic website called mspace. This website is not specifically for learning purposes. It is mainly a digital music library where people can search the music library semantically. But the University of Southampton implemented some part of semantics in his own website (http://www.ecs.soton.ac.uk). The main areas which university of Southampton cared to put some part of semantics on their online community are searching and displaying of information.

\subsubsection{Stanford University}

Stanford University has been studying and developing semantics and ontologies recently. They have been focusing more on $\mathrm{Al}$ and the semantics to improve their learning and building new applications. But unfortunately, they have not integrated the semantic completely with their website. The semantics are partly available for some of the academic resources of students and used only for searching mechanism. Stanford University was successful in their semantic research and they released a tool called 'Protégé' which will be used later to develop the ontologies. Despite the fact that they released a very powerful tool for ontology development, they have not been able to develop a complete semantic web to facilitate learning for their students.

\subsubsection{Knowledge Media Institute, the Open University}

$\mathrm{KMI}$ has a prestigious reputation for the development and use of knowledge-based technologies, especially in knowledge management, human-computer interaction and tools to support learning and teaching. It has an active interest in the Semantic Web, using Ontologies to develop portals and browsers and also working to develop Semantic WebServices.

\subsubsection{University of Maryland}

The University of Maryland has been working on a project specifically designed for improving learning and they have been making use of semantics. They have developed a website called mindswap (http://www.mindswap.org/). This site builds on a previous website that used a toolkit based on a web ontology language called SHOE. Mindswap mainly helps students about how to develop other semantic websites but this website itself contains some semantics technologies which helps the students in their research. 


\subsection{Critical Review of Existing Systems}

At the University of Southampton, it can be seen that they have been developing external semantic like mSpace but still they were a bit skeptical in implementing the whole semantics in their own website. They implement it in some parts of their websites like blogs, people and publications but it was not really helpful for students to improve their learning. Semantic on the website only helps searching for publications. There is not even the need for people to be a student to access some of it as they do not have a secure login system. Hence the website is not secure from unauthorized access.

At Stanford University, the same thing happened like that of the Southampton University. They released the 'Protégé' tools and they only implemented it in one part of their website which might help student when they are looking for researches and courses geographically related. Registration for students to help them is their work is also not visible.

At the University of Maryland, they developed a very good semantic website called the mindswap. It mainly contains information on the semantic technologies itself. Despite the fact that the semantics covers several aspects like the people, their papers, publications, etc, it is a poorly designed webpage. Semantics are sometimes not well used in some pages and it is not user-friendly. It mainly helps students on the semantic topic, it does not help as a general tool to improve the learning of students and also it focuses only on one faculty.

\section{METHODOLOGY AND PROPOSED SOLUTION}

The main problem in the current learning website is the lack of search possibilities. Social interaction is one of the factors which normally help to improve online learning as different students have different IQ's. Videos and multimedia is also an important factor which improves learning and these are not found in any of the available systems which we have considered. Hence, the new system should be able to solve the problem of social interaction and searching of resources and notes with multimedia.

\subsection{Proposed Solution}

To improve learning at the University Of Mauritius, part of a semantic website will be developed to help students in their studies. Web 2.0 searches for pages; Web 3.0 searches for information. Currently, most of the learning websites are of web 2.0 but this time part of semantic will be integrated for the following reasons:

- Semantic is the future web and research says that in the future all web 2.0 might be converted into semantic. As human beings, we want to develop tools that help us connect and communicate easily, develop relationships, and explore and understand the world around us, regardless of how technologically advanced we become.

- It will help the students better than the web 2.0 applications as many times when a student makes a search they do not have knowledge about what they are looking for and therefore might thus get inappropriate results. But with the semantic technologies, it will refine their search and will display the most appropriate result to them. It will also automatically direct them to some affiliates based on their search without any human intervention.

- A learning environment must be inspiring and trigger curiosity for the learning task. External links is therefore very important in a learning platform where students can browse for additional reading.

- On-line tests and quizzes will be done. Performance tracking for each student and each student can view their grading whenever they wish. This will help in their educational improvement by targeting higher grade.

\subsection{Tools to develop the semantic part}

Table 2: tools for semantic part

\begin{tabular}{|l|l|l|l|l|}
\hline Tools & User friendly & Robustness & Open-source & $\begin{array}{l}\text { Compatibility and } \\
\text { Issues }\end{array}$ \\
\hline $\begin{array}{l}\text { Topbraid } \\
\text { Composer }\end{array}$ & Nory powerful tool but \\
\hline
\end{tabular}




\begin{tabular}{|l|l|l|l|}
\hline Protege & Yes & $\begin{array}{l}\text { Some older versions } \\
\text { might contain bugs and } \\
\text { doesn't contain sparql } \\
\text { querying }\end{array}$ \\
\hline Jena & Yes & $\begin{array}{l}\text { Implemented in Java. It } \\
\text { might take more time to } \\
\text { develop it in php }\end{array}$ \\
\hline
\end{tabular}

One of the best tools to implement a semantic web is the Topbraid Composer but it is not free and open source and there we had to choose for Protégé which offers nearly same functionalities and also it is free.

The Protégé-OWL editor enables users to:

- $\quad$ Load and save OWL and RDF(S) ontologies.

- Modify and visualize classes, properties, and SWRL rules.

- Define logical class characteristics as OWL expressions.

- Accomplish reasoners such as description logic classifiers.

- Modify OWL individuals for Semantic Web markup.

\subsection{Description of the system}

To have a better online learning system at the University Of Mauritius, Semantic is introduced in a website which is going to help students of UoM for better learning. First of all, to get full access of the materials available on the website, students must register themselves and then log in using a username and password.

Students need to create an account for themselves and then enter some of their basic information like picture, status, etc. Their names, course, date of birth, etc will be displayed as when they register, and their respective data will already be available. There will be a small section in their profile where their performance, that is markings for test and exams, will be displayed. This performance will be entered by administers and students will not be able to edit it. The semantic part is when result of a student in a specific module is below the average level, the system will automatically provide him with some links related to the module without requiring the student to launch any search by him.

There will be another section where students will be able to browse data (papers, research, videos, etc) and can also launch a search. All the available courses, course description, course contents/ resources including lecture notes and videos to be downloaded will be available. Semantics will be used when students select a specific course. For example a student selects a module such as MIS (Management Information System), all the lectures of MIS will be made available to him. Moreover, some external links, useful papers, case studies and videos related to the MIS module will be made available without the need for the student to browse on the web to search notes by himself. Forums in which the module MIS was discussed will also be made available to him where he can view the forums and broaden his knowledge or clear misunderstandings. External links, resources, videos, forums and quizzes will also be automatically provided to students when they select a specific topic also. These will also be used to improve learning where students can interact with each other by posting questions and clarifying doubts where required.

There will also be a section where quizzes and assessments will be available to students. If a student has not successfully completed a unit or has got very poor grade, he/she will be advised to retake the unit before he/she can move to the next unit and semantics will automatically provide him/her with useful links to help $\mathrm{him} /$ her complete the unit to make sure the student improves on his overall performance. After the quizzes, answers will be provided and it can even redirect the students to some forums based on some specific key words addressed in the quizzes. If a quiz is made on MIS module and if a student has not scored some good scores in the quiz, it will provide him with additional links and will also spot some of the brightest students automatically based on their performances. It will then redirect him to these bright students where they can communicate via email or chat system will be implemented if time permits. Last but not least, there will be an administration section where administers and lecturers will be able to upload the notes, links, videos, quizzes and assessments for the students. 


\subsection{Generic Steps for the Development of Personalized Learning Systems}

Eventually what is proposed at the end of this research are generic steps for the development of Personalized Learning Environments / Systems for Learning Purposes.

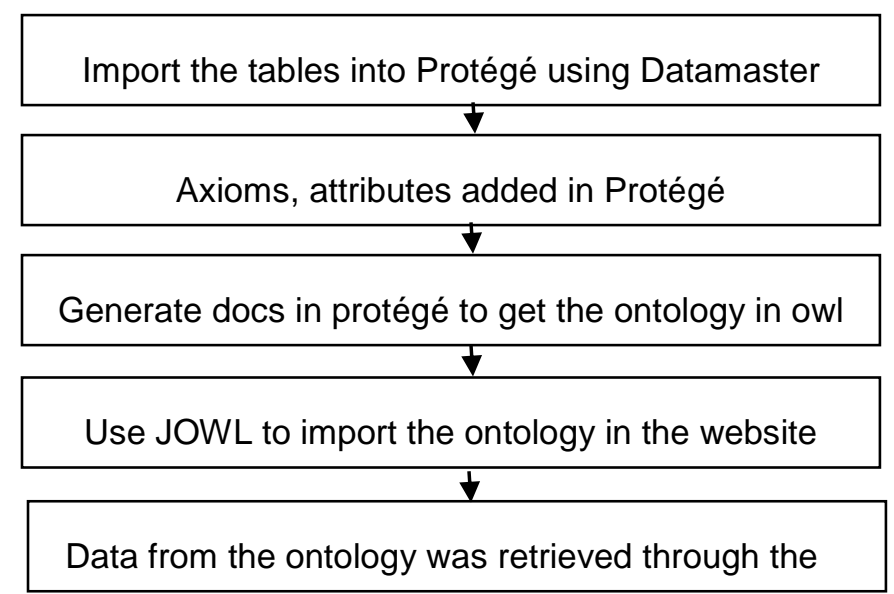

Figure 3: Steps to Design Semantic Part

\section{DISCUSSIONS AND CONCLUSION}

In this paper, a semantic model for web-based learning was described. This was made possible by the use of semantic web services and ontologies. The basic properties of the Semantic Web allow for meeting elearning requirements: quickness, just-in-time and pertinent learning. Course Materials can be annotated to be reused efficiently in other courses. Various researchers have observed that e-Learning, even when properly designed and meta-tagged, will not realize full re-usability without the full benefits from the Semantic Web. The use of Semantic in a learning website offers a number of advantages. It allows the reuse of established ontologies to unify descriptions of resources and enable data interoperability with other systems and data sets without in-depth knowledge of the application. It also simplifies the editing interface by allowing the user to interact with RDF. It also allows the use of content negotiation principles for ease of integration to 3rd party systems. Semantic annotation of resources by students and lecturers is possible. Exploring relationships between topics, resources, people and annotations, stored as RDF, for the purposes of building recommendation system and improving searching and saving time of student is possible. Future work will include the development of semantic web services for adaptive hypermedia and text mining.

\section{REFERENCE LIST}

[1] Angelova, G., Kalaydjiev, O., \& Strupchanska A. (2004). Domain ontology as a resource porividing adaptivity in e-learning, In Workshop on ontologies, semantics, and e-learning, on the move to meaningful internet systems (pp. 700-712)

[2] Berners-Lee, T., Hendler, J., \& Lassila, O. (2001). The semantic web, Scientific American, (pp. 34-43).

[3] Bittencourt, I., Costa, E., Soares, E., Pedro, A.(2008), Towards a new generation of webbased educational systems: the convergence between artificial and human agents, IEEE Multidisciplinary Engineering Education Magazine 3 (1) 17-24.

[4] Chen, W., Mizoguchi, R. (2004), Learner model ontology and leaner model agent, Cognitive Support for Learning - Imagining the Unknown 189-200

[5] Gruber, T. R. (1993). A translation approach to portable ontology specifications. Knowledge Acquisition, $5,199-220$

[6] Semantic web architecture[online], available from http://vinodkr.blogspot.com/ [accessed: 05 October 2015]

[7] Devedzic, Vladan. (2004). Education and the Semantic Web. International Journal of Artificial Intelligence in Education, 14, 39-65. 
[8] Protégé [online], available from http://protege.stanford.edu/[accessed: 05 October 2015]

[9] Aroyo, L., Dicheva, D.(2004), The new challenges for e-learning: the educational semantic web, Educational Technology and Society, 7 (4), pp. 59-69

[10] Sosnovsky, S., Mitrovic, A., Lee, D. , Brusilovsky, P., Yudelson, M., Brusilovsky, V., Sharma, D. (2008), Towards integration of adaptive educational systems: mapping domain models to ontologies, in: D. Dicheva, A. Harrer, R. Mizoguchi, (Eds.) Proceedings of 6th International Workshop on Ontologies and Semantic Web for E-Learning (SWEL'2008) in Conjunction with ITS'2008. Montreal, Canada.

[11] Yeo, J., Tan, S.C and Lee, Y.J. (2006), A learning journey in problem-based learning, in: ICLS: Proceedings of the 7th International Conference on Learning Sciences.

[12] Berners-Lee, T., Hendler,J and Lassila, O.(2001), The semantic web. A new form of web content that is meaningful to computers will unleash a revolution of new possibilities, Scientific American 284 (5) 3443.

[13] He, Y., Hiu, S. and Quan, T. (2009), Automatic summary assessment for intelligent tutoring systems, Computers and Education, 53, pp 890-899

[14] Zeng, Q., Zhao, Z., Liang, Y. (2009), Course ontology-based user's knowledge requirement acquisition from behaviors within e-learning systems,Computers and Education, 53 (2009), pp. 809-818

[15] Radenkovic, S.,Krdzavac, N., Devedzic, V. (2009)An assessment system on the semantic web,Annals of Information Systems, 6, pp. 177-200

[16] Bittencourt, I., Costa, E., Silva, M., Soares, E. (2009)A computational model for developing semantic web-based educational systems, Knowledge-Based Systems, 22 (2009), pp. 302-315 